\title{
Effect of urea on biomimetic aggregates
}

F.H. Florenzano and M.J. Politi
Departamento de Bioquímica and Laboratório Interdepartamental de Cinética Rápida, Instituto de Química, Universidade de São Paulo, 05508-900 São Paulo, SP, Brasil

\section{Correspondence \\ M.J. Politi \\ Instituto de Química, USP \\ Av. Lineu Prestes, 748 \\ 05508-900 São Paulo, SP \\ Brasil}

Presented at the 25th Anniversary Symposium of the Departamento de Bioquímica, Instituto de Química, Universidade de São Paulo, December 6-8, 1995, São Paulo, SP, Brasil.

Research supported by FAPESP, CNPq, CAPES, PADCT and FINEP

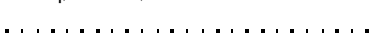

Received June 23, 1996

Accepted November 25, 1996 $\ldots \ldots \ldots \ldots \ldots \ldots \ldots$

\begin{abstract}
The effect of urea on biomimetic aggregates (aqueous and reversed micelles, vesicles and monolayers) was investigated to obtain insights into the effect of the denaturant on structured macromolecules. Direct evidence obtained from light scattering (static and dynamic), monolayer maximum isothermal compression and ionic conductivity measurements, together with indirect evidence from fluorescence photodissociation, fluorescence suppression, and thermal reactions, strongly indicates the direct interaction mechanism of urea with the aggregates. Preferential solvation of the surfactant headgroups by urea results in an increase in the monomer dissociation degree (when applied), which leads to an increase in the area per headgroup and also in the loss of counterion affinities.
\end{abstract}

\section{Introduction}

Amphiphiles, in general, present easily recognizable hydrophobic and hydrophilic regions, a feature not shared by most biomacromolecules. The effect of denaturants on the native conformation of proteins and nucleic acids has helped to firmly establish the involvement of the 3D structure of the macromolecule in biological activity (1). Denaturation mechanisms for various additives such as alcohols, surfactants, and urea, however, are still a matter of controversy (29). In addition, there is no comprehensive theory or model for the dynamics and structure of liquid water, the biological medium. Within this context, protein denaturation by urea, for example, has been analyzed within the framework of two opposite theories, i.e., direct interaction with the macromolecule or an indirect effect via rupture of the 3D network of liquid water.

Given the straightforward nature of simple surfactants and the various macromolecular aggregates which can be formed, the effect of urea upon surfactant mixtures has been investigated to determine the physicochemical origins of its action (10-16). In the present article, we will review the progress made by our research group towards the understanding of what urea does to aqueous solutions (complete details of the data presented here can be found in the original publications cited in Refs. 17-20). Data on the effects of urea on photoacid excited-state dissociations and ground-state reassociations in bulk water and in biomimetic aggregates (Figure 1) showed how the dynamic/solvatophobic of water and ionic diffusive motions are altered. Insoluble monolayer compression isotherms of various types of amphiphiles with urea added to the aqueous subphase demonstrated the binding of the additive to the interface and its ability to change the electroionic properties of the monolayer. Studies focusing on the behavior of electro- 
lytes on micellar and vesicular aggregates with urea in bulk water revealed the preferential sorption of the additive at the interface, altering, as is the case for monolayers, the ionic properties of the aggregates. Data are discussed in terms of the direct interaction mechanism and shed light on the control which can be exerted on surfactant interfaces by simple additives such as urea.

In Figure 1 typical macromolecular aggregates formed by amphiphile association are presented. These aggregates, also defined as biomimetic models or biomimetic aggregates, are powerful systems used to mimic selected properties of the biological media since several parameters are easier to modify and control. For example, the establishment of passive diffusion permeability coefficients of various ions through cell membranes was possible by determining them in a closed bilayer shell (vesicle) made with the appropriate lipids (1).

For the sake of clarity, the results on the effect of urea on surfactant aggregates re- viewed here are subdivided into the various classes of aggregates studied. The order of presentation is reversed micelles, monolayers, aqueous micelles and vesicles.

\section{Reversed micelles}

Reversed micelles (RM) are surfactant solutions in low polarity organic solvents (hexane, isooctane, $\mathrm{CCl}_{4}$ ) with water dispersed (when added) in the so-called aqueous core (Figure 1). For example, with the best characterized RM-forming amphiphile, sodium bis-ethylhexylsulfosuccinate (AOT), it is possible to dissolve up to $\sim 10 \%$ water ( $\mathrm{v} /$ $\mathrm{v})$ in $0.1 \mathrm{M}$ AOT in isooctane. AOT/RM with volume fractions $\left(\phi_{\mathrm{w}}\right) \leq 0.2$ are spherical aggregates (water droplets) which are usually characterized by the molar ratio between water and surfactant $\left(\mathrm{w}=\left[\mathrm{H}_{2} \mathrm{O}\right] /\right.$ [AOT]) (21).

The addition of urea to AOT/cyclohexane/water reversed micelles leads to an increase in the observed hydrodynamic radii

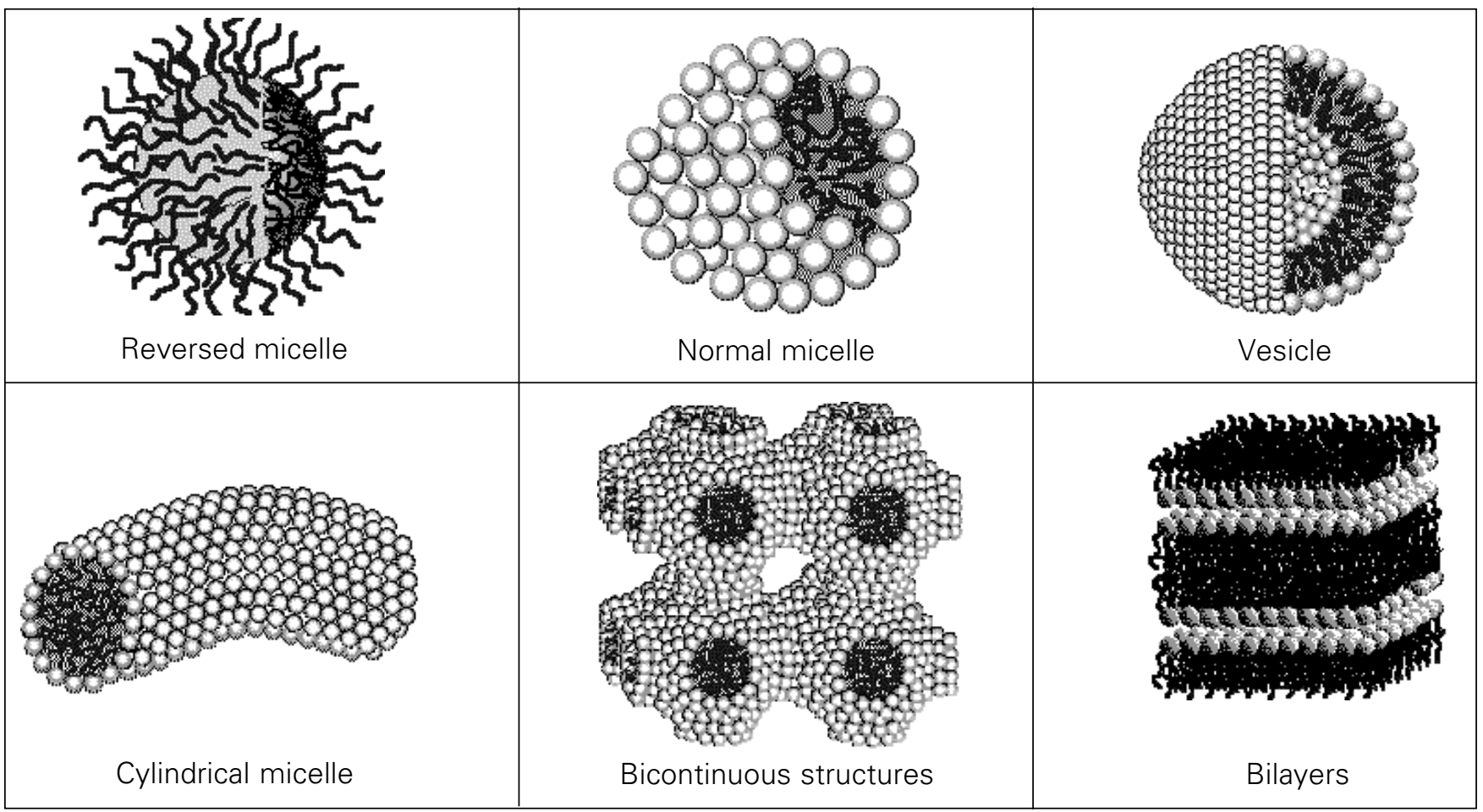

Figure 1 - Typical biomimetic aggregates. 
(Rh) (Figure 2) determined by Quasi-Elastic-Light-Scattering (QELS) (17). Clearly for the same $w, R h$ increased appreciably with the amount of urea in the aqueous microphase (note that the solubility of urea in the organic phase, hexane, is zero) (Figure 2). This increase may be due to actual micellar growth or simply to droplet juxtaposition, i.e., clustering.

In parallel, a large increase in the ionic conductivity $(\kappa)$ percolation threshold with relatively low RM volume fractions $\left(\phi_{\mathrm{w}}\right)$ was observed with the increase in urea concentration (Figure 3). Interestingly, by varying the temperature for fixed $\phi_{\mathrm{w}}$ 's an unusual decrease in the solution ionic conductance for $\phi_{\mathrm{w}} \sim 0.03$ with $5 \mathrm{M}$ urea was observed (Figure 4) which is strong evidence for RM declusterization with temperature. Recent X-ray and static-light-scattering (SLS) data (18) confirmed the hypothesis of RM clustering as a function of added urea, i.e., weightaveraged molecular weights remained invariant and scattering intensities were in agreement with discrete RM units. The introduction of urea into the aqueous pool of RM therefore enhances the intermicellar attractive term making a sticking contribution to $\mathrm{RM}$ aggregation without disturbing the discrete nature of individual droplets.

The solvatophobic properties of the AOT/ RM hydrophilic core (water plus urea) can be inferred by examining the effects on the photoacid properties of the triple negative charged probe (8-hydroxy-1,3,6-pyrenetrisulfonate, pyranine) (22-24). Photoacids are compounds whose excited state presents a stronger $\mathrm{pK}_{\mathrm{a}}$ and whose lifetime is long enough for the occurrence of prototropism. Light excitation leads therefore to $\mathrm{H}^{+}$dissociation when starting with a solution containing the undissociated ground-state species. For pyranine it is established that photoacid behavior occurs in water, aqueous urea, and only in a few other highly polar and protic media (25). Since this probe is relatively hydrophilic and bears the same charge

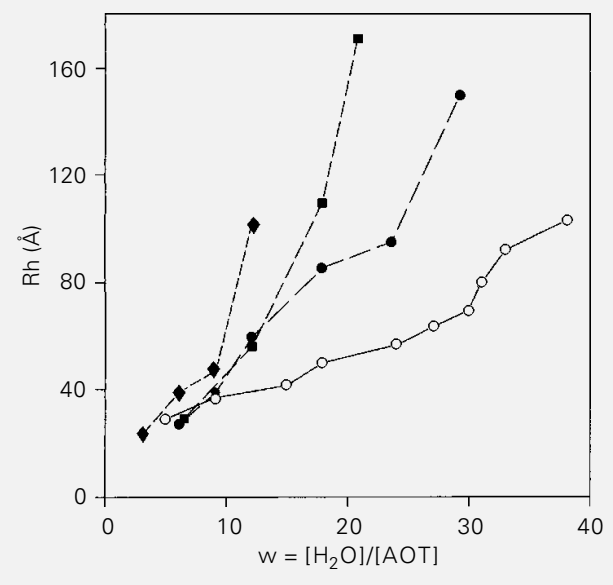

Figure 2 - Hydrodynamic radii (Rh) of AOT/hexane/water reversed micelles as a function of w. $[\mathrm{AOT}]=0.1 \mathrm{M}$; [urea] $=0(0)$; $3 \mathrm{M}(\bullet) ; 5 \mathrm{M}(\bullet) ; 10 \mathrm{M}(\bullet) . \mathrm{T}=$ $25^{\circ} \mathrm{C}$

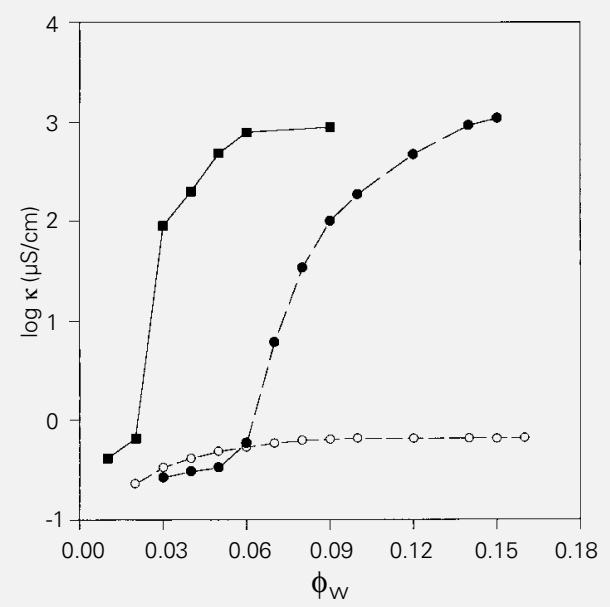

Figure 3 - Electric conductance $(\kappa)$ as a function of volume fraction $(\phi)$ in the system AOT/hexane/water/urea. [Urea] = $0 \mathrm{M}(O)$; $3 \mathrm{M}(\bullet) ; 5 \mathrm{M}(\mathbf{\square}) . \mathrm{T}=25^{\circ} \mathrm{C}$.

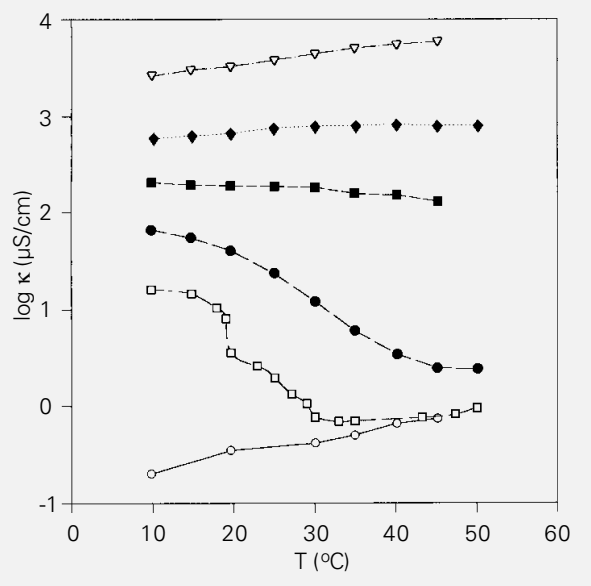

Figure 4 - Electric conductance ( $\kappa)$ as a function of temperature. [Urea] $=5 \mathrm{M} . \phi_{\mathrm{W}}=0.02(0) ; 0.03$ $(\square) ; 0.04(\bullet) ; 0.076(\bullet) ; 0.1(\bullet)$; $0.2(\nabla) . w=10$. 
Figure 5 - Proton association rate constants (kon) of $\mathrm{PO}^{-}$in AOT reversed micelles at various water and urea/water concentrations (w). $[\mathrm{AOT}]=0.1 \mathrm{M}$ and [urea] $=0 \mathrm{M}(0), 3 \mathrm{M}(\bullet), 5 \mathrm{M}$ $(\mathbf{\square}), 10 \mathrm{M}(\bullet)$

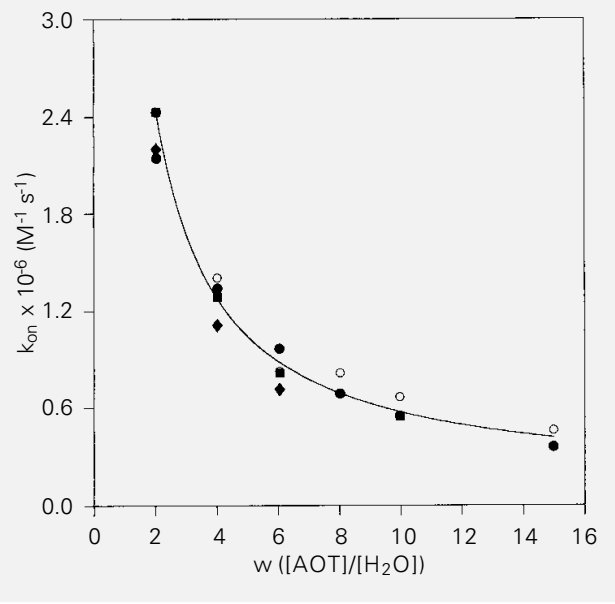

as the interface, it will reside in the interior of the aqueous pool (26). The increase in green fluorescence (emission of the excitedstate pyranine-conjugated base) as a function of the amount of the hydrophilic core added to the RM (or equivalently the decrease in the blue one, emission of the undissociated species) therefore can be related to the capacity of the aqueous pool to solvate the proton, $\mathrm{H}^{+}$. Emission spectra of pyranine in RM as a function of $\mathrm{w}$ in the presence or absence of urea are indistinguishable (data not shown) (17). In contrast, monitoring the recovery towards the pre-pulse level of pyranine species after a very short and strong laser pulse $(23,24,27)$ provides information on the diffusion of $\mathrm{H}^{+}$into the specific microenvironment. Again, the addition of urea to the aqueous pool does not affect the tran-

Table 1 - $A_{0}$ values $(\AA)$ for DODA monolayers in the absence and presence of $2 \mathrm{M}$ urea and $1 \mathrm{mM} \mathrm{NaX}$ $\left(\mathrm{X}^{-}=\mathrm{F}^{-}, \mathrm{Cl}^{-}, \mathrm{Br}^{-}, \mathrm{Ac}^{-}, \mathrm{NO}_{3}^{-}\right.$, and $\left.\mathrm{OH}^{-}\right)$at $\mathrm{T}=20,25$, and $30^{\circ} \mathrm{C}$.

\begin{tabular}{|c|c|c|c|c|c|c|}
\hline \multirow[t]{2}{*}{ Anion } & \multicolumn{2}{|c|}{$20^{\circ} \mathrm{C}$} & \multicolumn{2}{|c|}{$25^{\circ} \mathrm{C}$} & \multicolumn{2}{|c|}{$30^{\circ} \mathrm{C}$} \\
\hline & - & Urea & - & Urea & - & Urea \\
\hline $\mathrm{Br}^{-}$ & 54.5 & 116.2 & 56.3 & 115.7 & 64.5 & 112.7 \\
\hline $\mathrm{Cl}^{-}$ & 62.6 & 103.5 & 70.3 & 105.4 & 80.7 & 109.5 \\
\hline $\mathrm{F}^{-}$ & 85.4 & 111.2 & 83.6 & 112.2 & 89.6 & 118.4 \\
\hline $\mathrm{Ac}^{-}$ & 86.6 & 105.4 & 86.9 & 105.5 & 91.9 & 112.1 \\
\hline $\mathrm{NO}_{3}^{-}$ & 60.5 & 92.2 & 65.4 & 95.5 & 75.3 & 100.3 \\
\hline $\mathrm{OH}^{-}$ & 86.7 & 103.4 & 83.7 & 104.0 & 90.6 & 107.4 \\
\hline
\end{tabular}

siently imposed $\mathrm{H}^{+}$pulse compared to the pure water core (Figure 5), thus demonstrating the effect of the additive at the interface.

\section{Monolayers}

Monolayers are another biomimetic system composed of a single layer of an insoluble surfactant at the interface of two unmiscible phases, usually air-water. Monolayers can be compressed or expanded with a hydrophilic movable barrier and parameters such as surface pressure $\left(\Phi=\gamma_{0}-\gamma\right.$, where $\gamma_{0}$ and $\gamma$ are the surface tension in the absence and presence of surfactant), surface potential, and others are measured against the area per surfactant $(28,29)$.

The direct interaction of urea was demonstrated by monolayer studies on a Langmuir-Blodgett balance. Increasing the urea concentration in the subphase of anionic and cationic monolayers (carboxylate and quaternary ammonium surfactant headgroups, respectively) was accompanied by an enlargement in the monomer minimum area $\left(\mathrm{A}_{0}=\right.$ area occupied per surfactant at a compression just before monolayer collapse) and also by the decrease or even loss in the ionic affinity series (Table 1). The increase in $\mathrm{A}_{0}$ is a direct manifestation of two effects: preferential sorption of urea at the interface and increase in the monolayer ionization degree resulting in an extra-electrostatic repulsive term which hinders the approximation between surfactants. Since effects on $\mathrm{A}_{0}$ and the ion affinity order occur at urea concentrations as low as $\sim 0.1 \mathrm{M}$, the most plausible interaction mechanism is sorption of urea at the interface, squeezing out water molecules which had been solvating the amphiphiles (19). It is tempting to associate the increase in the monolayer ionic dissociation degree with an increase in the local dielectric constant $(\varepsilon)$ due to replacement of water by urea $\left(\varepsilon_{\text {water }}=80\right.$ and $\varepsilon_{\text {urea } 5 \mathrm{M}}$ = 95) (2). 


\section{Aqueous micelles and vesicles}

Aqueous micelles and vesicles are the systems most frequently investigated in this field. Micelles have been extensively studied in terms of their effects on chemical reactivity (21), and vesicles due to their compartmentalization properties and therefore for their ability to mimic membrane properties. Micelles are formed by the spontaneous aggregation of amphiphiles in solution above certain critical concentrations and their properties will depend on the type of surfactant monomer employed. In contrast, vesicles are, in general, meta-stable suspensions of double-tail surfactants (lecithin, dialkyl-ammonium, etc.) formed by vortexing, ultrasonication, and other methods (30).

The effects of urea on ionic aqueous micellar and vesicular aggregates were investigated to obtain a more complete picture of the macromolecular systems depicted in Figure 1. The following biomimetic aggregates were studied: cetyltrimethylammonium bromide $(\mathrm{CTABr})$ and chloride $(\mathrm{CTACl})$, and hexadecyldimethylammonium propanesulfonate (HPS) micelles and dialkyldimethylammonium chloride (HERQUAT) vesicles. These three systems share a common quaternary group located at the interface region with the cationic monolayers and are expected to be subject to the same favorable interaction with urea. Studies were directed at the examination of the two following aspects: i) effects on the formation properties of the aggregates, and ii) ion association selectivity and exchange at the interface (20).

The addition of urea clearly diminished the hydrophobic interaction by increasing the solubility of monomers and, therefore, delaying the onset for micelle formation (Table 2). Furthermore, simple micelle dissociation degree $(\alpha)$ calculations (ratio of slopes before and after the critical micellar concentration, CMC) from ionic conductivity measurements showed a trend towards

\begin{tabular}{|c|c|c|c|}
\hline Surfactant & $\begin{array}{c}\text { Urea } \\
\text { concentration } \\
\text { (M) }\end{array}$ & $\mathrm{CMC}$ & $\alpha$ \\
\hline \multirow[t]{5}{*}{ HPS } & 0 & $20.9 \mu \mathrm{M}$ & - \\
\hline & 0.50 & $22.8 \mu \mathrm{M}$ & - \\
\hline & 1.00 & $26.8 \mu \mathrm{M}$ & - \\
\hline & 2.00 & $37.3 \mu \mathrm{M}$ & - \\
\hline & 5.00 & $81.1 \mu \mathrm{M}$ & - \\
\hline \multirow[t]{5}{*}{ CTACl } & 0 & $1.60 \mathrm{mM}$ & 0.33 \\
\hline & 0.5 & $1.64 \mathrm{mM}$ & 0.36 \\
\hline & 1 & $1.68 \mathrm{mM}$ & 0.37 \\
\hline & 3 & $2.31 \mathrm{mM}$ & 0.42 \\
\hline & 5 & $3.03 \mathrm{mM}$ & 0.44 \\
\hline
\end{tabular}

higher $\alpha$ 's, i.e., to an increase in the area per monomer at the interface with urea which again points to a direct interaction of the denaturant with the interface. For the case of HERQUAT vesicles monitoring Rh by QELS and weight averaged molecular weight (or equivalently, mean aggregation numbers) by SLS as a function of incubation time (days) showed that introduction of urea into the preparation, although not inhibiting the formation of vesicles, certainly decreased its stability (Table 3 ). Vesicle growth may be a manifestation of interbilayer enhanced attractive contribution due to interfacial sorption of urea.

Table 3 - Molecular weight and hydrodynamic radii of HERQUAT vesicles as a function of incubation time and urea concentration.

a Vesicles prepared in $2 \mathrm{mM} \mathrm{NaCl}$; ${ }^{b}$ vesicles prepared in $1 \mathrm{mM} \mathrm{NaCl}$; ${ }^{c}$ the accurancy of light-scattering determinations is about $5 \%$.

\begin{tabular}{|c|c|c|c|c|c|c|}
\hline \multirow{2}{*}{$\begin{array}{l}\text { Time } \\
\text { (days) }\end{array}$} & \multicolumn{3}{|c|}{ Molecular weight ${ }^{c} \times 10^{-6} \mathrm{~g} / \mathrm{mol}$} & \multicolumn{3}{|c|}{ Hydrodynamic radii (Å) } \\
\hline & Watera & 3 M Urea ${ }^{a}$ & 5 M Ureab & Water & 3 M Urea & 5 M Urea \\
\hline 0 & 4.02 & 3.65 & 6.25 & 154.0 & 158.0 & 163.0 \\
\hline 1 & 4.93 & 4.59 & 8.73 & 156.5 & 167.0 & 177.0 \\
\hline 7 & 6.50 & 7.37 & 14.87 & 169.0 & 191.5 & 218.0 \\
\hline 13 & 7.03 & 8.30 & 12.19 & 177.5 & 214.5 & 234.0 \\
\hline 20 & 8.54 & 10.30 & 13.15 & 179.0 & 223.5 & 249.0 \\
\hline 34 & 8.66 & 11.25 & 10.71 & 185.5 & 238.5 & 270.5 \\
\hline
\end{tabular}


Figure 6 - Stern-Volmer projection for ß-NBN $(4.14 \mu \mathrm{M})$ incorporated into $40 \mathrm{mM} \mathrm{CTACl} \mathrm{mi-}$ celles in the absence $(O)$ and presence $(\bullet)$ of $3 \mathrm{M}$ urea as a function of $\mathrm{NaBr}$ concentration. Solid lines are from data simulation according to the PPIEM formalism.

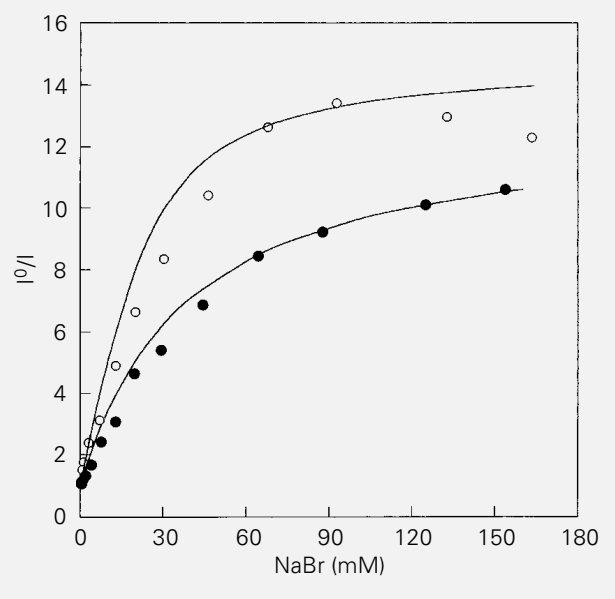

The effect of ion condensation and exchange on the systems studied by differential bromide and chloride fluorescence suppression of N-butyl-2,3-naphthalimide (BNBN) (Stern-Volmer suppression constants, $\mathrm{Ksv}=40$ and $28 \mathrm{M}^{-1}$ for $\mathrm{Br}^{-}$and 1 and 0.26 $\mathrm{M}^{-1}$ for $\mathrm{Cl}^{-}$in water and with $3 \mathrm{M}$ urea, respectively) (Figure 6) showed that the higher affinity of bromide over chloride for these interfaces was highly reduced in the presence of urea. For example, for CTACl submitted to the addition of $\mathrm{NaBr}$, the ionic exchange constant using the PPIEM formalism (31) decreased from $~ 4.5$ in bulk water to $\sim 1.1$ in the presence of $3 \mathrm{M}$ urea. This effect is once again a direct manifestation of a local, direct effect of urea at the interface.

\section{Conclusion}

The observed effects of urea on structural and dynamic properties of the biomimetic systems investigated indicate the localized action of the additive. For example, the enhancement of $\mathrm{A}_{0}$ of ionic monolayers with subphase urea concentrations of the order of $0.1 \mathrm{M}$ cannot be attributed to a bulk solvent disorganization phenomenon. Overall, the evidence presented strongly supports the direct interaction mechanism of urea, and, furthermore, it demonstrates how the properties of surfactant aggregates can be modulated with the use of an inert additive such as urea.

\section{Acknowledgments}

We wish to express our deep gratitude to our co-workers Prof. Hernan Chaimovich and Dr. Iolanda Cuccovia without whose help this work could not have been conducted. We also wish to acknowledge students and collaborators involved in the various projects: Dr. C.L. Costa, Mr. O.L. Brino, Mr. L.G. Santos, Ms. S.M.B. Souza and Ms. M.V. Scarpa. We also wish to thank the organizing committee of the Biochemistry Department 25th Year Symposium.

\section{References}

1. Voet D \& Voet JD (1995). Biochemistry. 2nd edn. John Wiley and Sons, New York.

2. Franks F (1973). Water: A Comprehensive Treatise. 1st edn. Plenum Press, New York.

3. Robinson DR \& Jencks WP (1965). The effect of compounds of the urea-guanidinium class on the activity coefficient of acetyltetraglycine ethyl ester and related compounds. Journal of the American Chemical Society, 87: 2462-2468.

4. Liepinsh E \& Otting G (1994). Specificity of urea binding to proteins. Journal of the American Chemical Society, 116: 96709674.
5. Baglioni P, Ferroni E \& Kevan L (1990). Electron spin echo modulation study of sodium dodecyl sulfate and dodecyltrimethylammonium bromide micellar solutions in the presence of urea: evidence of urea interaction at the micelle surface. Journal of Physical Chemistry, 94: 42964298

6. Kang YS, McManus HJD \& Kevan L (1992). An electron magnetic resonance study on the photoionization of $\mathrm{N}$ alkylphenothiazines in dioctadecyldimethylammonium chloride vesicles: the effect of urea, 1,3-dimethylurea, 1,3diethylurea, and 1,1',3,3'-tetramethylurea. Journal of Physical Chemistry, 96: 1005510060.
7. Kuharski RA \& Rossky PJ (1984). Molecular dynamics of solvation in urea-water solution. Journal of the American Chemical Society, 106: 5786-5793.

8. Nandi PK \& Robinson DR (1984). Effects of urea and guanidine hydrochloride on peptide and nonpolar groups. Biochemistry, 23: 6661-6668.

9. Wetlaufer DB, Malik SK, Stoller L \& Coffin $R L$ (1964). Nonpolar group participation in the denaturation of proteins by urea and guanidinium salts: model compound studies. Journal of the American Chemical Society, 86: 508-514. 
10. Benjamin L (1966). Calorimetric study of the effect of urea, guanidium salts and alcohols on the micellization of dimethyldecylamine. Journal of Colloid and Interface Science, 22: 386-390.

11. Corkill JM, Goodman JF, Harrold SP \& Tate JR (1967). Effect of urea on thermodynamics of micellization. Transactions of the Faraday Society, 63: 240-246.

12. Bruning $W \&$ Holtzer $A$ (1961). The effect of urea on hydrophobic bonds: the critical micelle concentration of $\mathrm{n}$-dodecyltrimethylammonium bromide in aqueous solutions of urea. Journal of the American Chemical Society, 83: 4865-4866.

13. Jha R \& Ahluwalla JC (1993). Thermodynamics of micellization of some decyl poly(oxyethylene glycol) ethers in aqueous urea solutions. Journal of the Chemical Faraday Society Transactions, 89: 3465-3469.

14. Emerson MF \& Holtzer A (1967). The hydrophobic bonds in micellar systems. Effects of various additives on the stability of micelles of sodium dodecyl sulfate and of n-dodecyltrimethylammonium bromide. Journal of Physical Chemistry, 71: 3320-3330.

15. Almgren $M$ \& Swarup $S$ (1983). Size of sodium dodecyl sulfate micelles in the presence of additives. Journal of Colloid and Interface Science, 9: 256-266.
16. Mukerjee P \& Ray A (1963). The effect of urea on micelle formation and hydrophobic bonding. Journal of Physical Chemistry, 67: 190-192.

17. Amaral CLC, Brino $\mathrm{O}$, Chaimovich $\mathrm{H}$ \& Politi MJ (1992). Formation and properties of reversed micelles of AOT containing urea in the aqueous pool. Langmuir, 8: 2417-2421.

18. Amaral CLC, Itri R \& Politi MJ (1996). Structure determination of AOT/n-hexane/ water/urea reversed micelles by light and small angle $X$-ray scattering. Langmuir, 12: 4638-4643.

19. De Souza SMB, Chaimovich H \& Politi MJ (1995). Effects of urea on dioctadecyldimethylammonium monolayers. Langmuir, 11: 1715-1719

20. Florenzano FH, Santos LGC, Cuccovia IM, Scarpa MV, Chaimovich H \& Politi MJ (1996). Urea decreases anion selectivity in cationic micelles. Langmuir, 12: 11661171

21. Fendler JH (1982). Membrane-Mimetic Chemistry. 1st edn. Wiley-Interscience, New York.

22. Ireland JF \& Wyatt PAH (1976). Acid-base properties of electronically excited states of organic molecules. Advances in Physical Organic Chemistry, 12: 131-221.

23. Gutman $\mathrm{M}$ (1984). The $\mathrm{pH}$ jump: probing of macromolecules by a laser-induced, ultrashort proton pulse - theory and applications. Methods in Biochemical Analysis, 30: 1-103.
24. Gutman M (1986). Application of the laser-induced proton pulse for measuring the protonation rate constants of specific sites on proteins and membranes. Methods in Enzymology, 127: 522-538.

25. Politi MJ, Brandt O \& Fendler JH (1985). Ground and excited state proton transfer in reversed micelles. Polarity restrictions and isotopic effects. Journal of Physical Chemistry, 89: 2345-2354.

26. Politi MJ \& Chaimovich $\mathrm{H}$ (1986). $\mathrm{H}_{2} \mathrm{O}$ activity in reversed micelles. Journal of Physical Chemistry, 90: 282-287.

27. Politi MJ \& Fendler JH (1984). Laser pHjump initiated proton transfer on charged micellar surfaces. Journal of the American Chemical Society, 106: 265-273.

28. Hann RA (1990). Molecular structure and monolayer properties. In: Roberts G (Editor), Langmuir-Blodgett Films. Plenum Press, New York.

29. Gaines Jr GL (1966). Insoluble Monolayers at Liquid-Gas Interface. WileyInterscience, New York.

30. Szoka Jr F \& Papahadjopoulos D (1980). Comparative properties and methods of preparation of lipid vesicles (liposomes). Annual Review of Biophysics and Bioengineering, 9: 467-508.

31. Quina FH \& Chaimovich H (1979). Ion exchange in micellar solutions. 1. Conceptual framework for ion exchange in micellar solutions. Journal of Physical Chemistry, 83: 1844-1850. 